\title{
Pacific
}

Journal of

Mathematics

\section{ADJOINT LINEAR SYSTEMS ON A SURFACE OF GENERAL TYPE IN POSITIVE CHARACTERISTIC}

TOHRU NAKASHIMA 


\title{
ADJOINT LINEAR SYSTEMS \\ ON A SURFACE OF GENERAL TYPE IN POSITIVE CHARACTERISTIC
}

\section{TOHRU NAKASHIMA}

\begin{abstract}
Let $X$ be a minimal surface of general type defined over an algebraically closed field of positive characteristic $p$. For a given divisor $D$, we consider the spannedness properties of adjoint linear systems $|K+D|$ on $X$. Under some numerical conditions on $p$ and $D$, the failure of spannedness of $|K+D|$ implies the existence of divisors with special properties. This leads to the following result: Let $L$ be an ample line bundle and assume $p \geq 5$. Then $|m(K+L)|$ is base point free for $m \geq 2$ and very ample for $m \geq 3$. Our proof is based on a technique of Shepherd-Barron using unstable vector bundles.
\end{abstract}

1. Introduction. After Reider introduced a new method ([R]), many results have been obtained concerning adjoint linear systems on algebraic surfaces defined over an algebraically closed field of characteristic 0. Recently, Shepherd-Barron ([SB]) treated the positive characteristic case and obtained results on pluricanonical systems, improving the work of Ekedahl ([E]). He also showed Reider's analysis holds for surfaces of special type except the quasi-elliptic ones. His method is, as in $[\mathbf{R}]$, based on the theory of unstable vector bundles in the sense of Bogomolov.

In the present note we shall consider adjoint linear systems on a minimal surface of general type in characteristic $p$ and prove some results of Reider's type.

Let $X$ be a minimal surface of general type defined over an algebraically closed field $k$ of char $k=p>0$ and let $D$ be a nef divisor such that $D-K$ is nef and big. We shall prove the following

THEOREM 1. Let $X$ and $D$ be as above and let $d:=D^{2}$.

(i) Suppose that one of the following conditions holds:

(1) $p \geq 2, d \geq 5$ and $X$ is not uniruled,

(2) $p=3$ and $d \geq 12$,

(3) $p=5$ and $d \geq 6$,

(4) $p \geq 7$ and $d \geq 5$.

If $|K+D|$ has a base point, then there exists an effective divisor $\Delta$ such 
that

$$
\begin{array}{rll}
\text { either } & D \cdot \Delta=1, & \Delta^{2}=0, \\
\text { or } & D \cdot \Delta=0, & \Delta^{2}=-1 .
\end{array}
$$

(ii) Suppose that one of the following conditions holds:

( $\left.1^{\prime}\right) \quad p \geq 2, d \geq 10$ and $X$ is not uniruled,

(2') $p=3$ and $d \geq 30$,

(3') $p=5$ and $d \geq 13$,

(4') $p=7$ and $d \geq 11$,

$\left(5^{\prime}\right) \quad p \geq 11$ and $d \geq 10$.

If $|K+D|$ is not very ample, then there exists an effective divisor $\Delta$ such that

$$
\begin{aligned}
\text { either } & D \cdot \Delta=0, & \Delta^{2}=-1,-2, \\
\text { or } & D \cdot \Delta=1, & \Delta^{2}=-1,0, \\
\text { or } & D \cdot \Delta=2, & \Delta^{2}=0 .
\end{aligned}
$$

As a corollary of the above theorem, we obtain the following result on pluri-adjoint systems:

Corollary 2. Let $L$ be an ample divisor on $X$.

(i) Suppose that one of the following conditions holds:

(1) $p \geq 2, m \geq 2$ and $X$ is not uniruled,

(2) $p=3$ and $m \geq 3$,

(3) $p \geq 5$ and $m \geq 2$.

Then $|m(K+L)|$ is base point free.

(ii) Suppose that one of the following conditions holds:

( $\left.1^{\prime}\right) p \geq 2, m \geq 3$ and $X$ is not uniruled,

(2') $p=3$ and $m \geq 4$,

(3') $p \geq 5$ and $m \geq 3$.

Then $|m(K+L)|$ is very ample.

Proof. Apply the theorem to $D=(m-1) K+m L$.

2. Proof of the theorem. Let $p$ be a base point of $|K+D|$ in (i) (resp. $p, q$ be the points not separated by $|K+D|$ in (ii)) and let $\pi: \widetilde{X} \rightarrow X$ be the blowing up at $p$ in (i) (resp. at $p$ and $q$ in (ii)). Put $l:=\pi^{-1}(p), m:=\pi^{-1}(q)$, and $\widetilde{D}:=\pi^{*} D-2 l$ in (i) (resp. $\widetilde{D}:=\pi^{*} D-2(l+m)$ in (ii)).

Since we have $H^{1}\left(\widetilde{X}, \mathscr{O}_{\widetilde{X}}(-\widetilde{D})\right) \neq 0$, we have a nonsplit sequence on $\widetilde{X}$ :

$$
0 \rightarrow \mathscr{O}_{\widetilde{X}} \rightarrow E \rightarrow \mathscr{O}_{\widetilde{X}}(\widetilde{D}) \rightarrow 0
$$


Since $E$ satisfies the inequality $c_{1}(E)^{2}>4 c_{2}(E)$, by Theorem 1 in [SB], there exists a Frobenius map $F^{e}: \widetilde{X} \rightarrow \widetilde{X}$ and an exact sequence

$$
0 \rightarrow \mathscr{O}_{\widetilde{X}}\left(p^{e} \widetilde{D}-\Delta_{1}\right) \rightarrow \widetilde{E} \rightarrow \mathscr{I}_{Z} \otimes \mathscr{O}_{\widetilde{X}}\left(\Delta_{1}\right) \rightarrow 0 .
$$

Here $\widetilde{E}:=\left(F^{e}\right)^{*} E, Z$ is a 0 cycle and $\Delta_{1}$ is some effective divisor such that $p^{e} \widetilde{D}-2 \Delta_{1}$ is contained in the positive cone of $\widetilde{X}$. We write $\Delta_{1}=\pi^{*} \Delta+r l$ in (i) (resp: $\Delta_{1}=\pi^{*} \Delta+r l+s m$ in (ii)) where $r, s$ are some integers and $\Delta$ is an effective divisor on $X$.

If $p^{e}=1$, Reider's argument shows $\Delta$ satisfies the properties stated in the theorem (cf. $[\mathbf{R}]$ ). We shall show that the case $p^{e} \geq p$ never occurs under our assumption.

Suppose $p^{e} \geq p$. Then there is a purely inseparable covering $\rho: Y \rightarrow \widetilde{X}$ of $\operatorname{deg} \rho=p^{e}$ and we have the following estimates (cf. [SB]).

Lemma 3. Assume $d:=D^{2} \geq 5$ in (i) (resp. $d \geq 10$ in (ii)). Then

$$
D \cdot \Delta \leq\left(\frac{d}{2}-\sqrt{\frac{d^{2}}{4}-d}\right) p^{e}
$$

and

$$
\chi\left(\mathscr{O}_{Y}\right) \geq\left(\chi\left(\mathscr{O}_{X}\right)+\frac{p^{e}-1}{12}\left[\left(2 p^{e}-1\right) \widetilde{D}^{2}-3 \widetilde{D} \cdot K_{\widetilde{X}}\right]\right) p^{e}
$$

Since both $D$ and $D-K$ are nef and big, the Hodge index theorem yields $K \cdot D \leq d-3$ and $K^{2} \leq d-5$ in (i) (resp. $K^{2} \leq d-6$ in (ii)). By these estimates, we have

$$
\begin{aligned}
\omega_{Y} \cdot \rho^{*} \pi^{*} D & =2\left(p^{e}-1\right) D \cdot \Delta+p^{e}\left(K \cdot D-\left(p^{e}-1\right) D^{2}\right) \\
& \leq 2\left(p^{e}-1\right)\left(\frac{d}{2}-\sqrt{\frac{d^{2}}{4}-d}\right)+p^{e}\left(d-3-\left(p^{e}-1\right) d\right) \\
& =\left(d-3-\left(p^{e}-1\right) \sqrt{d^{2}-4 d}\right) p^{e}
\end{aligned}
$$

Thus we obtain $\omega \cdot \rho^{*} \pi^{*} D<0$. Therefore $Y$ is ruled and hence $X$ is uniruled. Let $q(X)$ be the irregularity of $X$. Then by Lemma 34 in [SB], we have $\chi\left(\mathscr{O}_{Y}\right) \leq 1-q(X) \leq 1$. Assume we are in the case 
(i). Then Corollary 30 and Proposition 35 in the same paper yield

$$
\begin{aligned}
\chi\left(\mathscr{O}_{Y}\right) & \geq\left(\chi\left(\mathscr{O}_{X}\right)+\frac{p^{e}-1}{12}\left[\left(2 p^{e}-1\right) \widetilde{D}^{2}-3 \widetilde{D} \cdot K_{\widetilde{X}}\right]\right) p^{e} \\
& \geq\left(-\frac{K^{2}}{10}+\frac{p^{e}-1}{12}\left[\left(2 p^{e}-1\right)\left(D^{2}-4\right)-3(D \cdot K+2)\right]\right) p^{e} \\
& \geq\left(-\frac{d-5}{10}+\frac{p^{e}-1}{12}\left[\left(2 p^{e}-1\right)(d-4)-3(d-3)-6\right]\right) p^{e} .
\end{aligned}
$$

Similarly in the case (ii) we obtain

$$
\chi\left(\mathscr{O}_{Y}\right) \geq\left(-\frac{d-6}{10}+\frac{p^{e}-1}{12}\left[\left(2 p^{e}-1\right)(d-8)-3(d-3)-12\right]\right) p^{e} .
$$

However, under the assumption that one of (1) to (4) (resp. $\left(1^{\prime}\right)$ to $\left.\left(5^{\prime}\right)\right)$ holds, we have $\chi\left(\mathscr{O}_{Y}\right)>1$. This is a contradiction and hence the theorem is proved.

\section{REFERENCES}

[E] T. Ekedahl, Canonical models of surfaces of general type in positive characteristic, Publ. Math. I.H.E.S., 67 (1988), 97-144.

[R] I. Reider, Vector bundles of rank 2 and linear systems on algebraic surfaces, Ann. of Math., 127 (1988), 309-316.

[SB] N. I. Shepherd-Barron, Unstable vector bundles and linear systems on surfaces in characteristic $p$, preprint.

Received March 18, 1991.

Tokyo Metropolitan University

TOKYO 192-03, JAPAN 


\section{CONTENTS}

G. D. Anderson, M. K. Vamanamurthy, and M. Vuorinen, Inequalities for quasi-

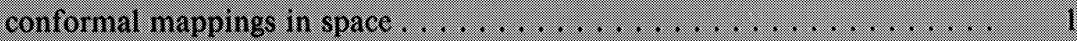

T. Bhattacharya, A nonexistence result for the $n$-Laplacian . . . . . . . . . . . . 19

J. A. Cima, K. Stroethoff, and K. Yale, Bourgain algebras on the unit disk . . . . . 27

J. A. Fridy and C. Orhan, Lacunary statistical convergence . . . . . . . . . . . . . . 43

D. Grenier, On the shape of fundamental domains in $\mathrm{GL}(n, \mathbf{R}) / \mathrm{O}(n) \ldots \ldots . . .53$

B. Jiang and J. Guo, Fixed points of surface diffeomorphisms . . . . . . . . . . . 67

P. Lejarraga, The moduli of rational Weierstrass fibrations over $\mathbf{P}^{\prime}$ : singularities 91

G. J. Martin, On discrete isometry groups of negative curvature . . . . . . . . . . 109

T. Nakashima, Adjoint linear systems on a surface of general type in positive characteristic . . . . . . . . . . . . . . . . . . . . . . . . . 129

B. Ralph, A homotopy transfer for finite group actions $\ldots \ldots \ldots \ldots \ldots \ldots \ldots$

Y. Rong, Maps between Seifert fibered spaces of infinite $\pi_{1} \ldots \ldots \ldots \ldots \ldots, 143$

J.-Y. Shi, Some numeric results on root systems . . . . . . . . . . . . 155

E. Spanier, Singular homology and cohomology with local coefficients and duality

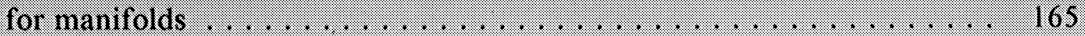




\section{PACIFIC JOURNAL OF MATHEMATICS}

Volume $160 \quad$ No. $1 \quad$ September 1993

Inequalities for quasiconformal mappings in space

GLEN DOUglas ANDERson, MaVina KRISHNa VAMANAMURThy and MATTI VUORINEN

A nonexistence result for the $n$-Laplacian

TILAK BHATTACHARYA

Bourgain algebras on the unit disk

Joseph A. Cima, Karel M. Stroethoff and Keith Yale

Lacunary statistical convergence

JOHN ALBERT FRIDY and CIHAN ORHAN

On the shape of fundamental domains in $\operatorname{GL}(n, \mathbf{R}) / \mathrm{O}(n)$

DOUGLAS MARTIN GRENIER

Fixed points of surface diffeomorphisms

BOJU JIANG and JIANHAN GUO

The moduli of rational Weierstrass fibrations over $\mathbf{P}^{1}$ : singularities

PABLO LEJARRAGA

On discrete isometry groups of negative curvature

GAVEN MARTIN

Adjoint linear systems on a surface of general type in positive characteristic

TOHRU NAKASHIMA

A homotopy transfer for finite group actions

WILLIAM J. RALPH

Maps between Seifert fibered spaces of infinite $\pi_{1}$

YONGWU RONG

Some numeric results on root systems

J. Y. SHI

Singular homology and cohomology with local coefficients and duality for manifolds

EDWIN SPANIER 